\title{
Concussion in professional football
}

\section{Summary of the research conducted by the National Football League's Committee on Mild Traumatic Brain Injury}

\author{
Elliot J. Pellman, M.D., and David C. Viano, Dr. Med., Ph.D. \\ Mild Traumatic Brain Injury Committee, National Football League, New York, New York; \\ ProHEALTH Care Associates, Lake Success, New York; \\ and ProBiomechanics, Bloomfield Hills, Michigan
}

\begin{abstract}
$\checkmark$ In 1994 the National Football League (NFL) initiated a comprehensive clinical and biomechanical research study of mild traumatic brain injury (TBI), a study that is ongoing. Data on mild TBIs sustained between 1996 and 2001 were collected and submitted by NFL team physicians and athletic trainers, and these data were analyzed by the NFL's Committee on Mild Traumatic Brain Injury. At the same time, analysis of game videos was performed for on-field mild TBIs to quantify the biomechanics involved and to develop means to improve the understanding of these injuries so that manufacturers could systematically improve and update their head protective equipment. The findings and analysis of the Committee have been presented in a series of articles in Neurosurgery.
\end{abstract}

\section{KEY WORDS • traumatic brain injury • sports-related concussion • neuropsychological assessment}

I N $1992 \mathrm{Al}$ Toon, who was a wide receiver for the New York Jets, was the first NFL player known to have retired because of postconcussion syndrome. ${ }^{2}$ The year after Mr. Toon's retirement, another player, Merrill Hoge of the Chicago Bears, retired because of the same problem. Commissioner Paul Tagliabue, team physicians, and many others raised questions: was this a new problem or a misdiagnosed or unrecognized one? Was this a statistical anomaly or the beginning of an epidemic?

It was decided that a rigorous, scientific approach was necessary to gather the data to answer these questions for this high-profile professional sports league. In 1994, Commissioner Tagliabue approved the creation of the NFL's Committee on Mild Traumatic Brain Injury. ${ }^{12}$ The Committee was composed of experts inside and outside the NFL. It was decided by the Committee that protection against injury as well as collection and analysis of injury data would be critical to the success of their mission. For the study, a reportable mild TBI was defined as a traumatically induced alteration in brain function manifested by an alteration of awareness and consciousness, including but not limited to an LOC, a "ding," a sensation of being dazed or stunned, a sensation of "wooziness" or "fogginess," a seizure or amnesic period, and by symptoms commonly associated with postconcussion syndrome, including persistent headaches, vertigo, lightheadedness, loss of balance, unsteadiness,

Abbreviations used in this paper: $\mathrm{CI}=$ confidence interval; ImPACT $=$ Immediate Postconcussion Assessment and Cognitive Testing; $\mathrm{LOC}=$ loss of consciousness; $\mathrm{mph}=$ miles per hour; $\mathrm{NFL}=$ National Football League; NOCSAE = National Operating Committee on Standards for Athletic Equipment; SI = severity index; TBI = traumatic brain injury. syncope, near-syncope, cognitive dysfunction, memory disturbances, hearing loss, tinnitus, blurred vision, diplopia, visual loss, personality change, drowsiness, lethargy, fatigue, and inability to perform usual daily activities. ${ }^{10}$ The research summarized here was developed, supervised, and completed in response to the stated goals of the NFL's Committee on Mild Traumatic Brain Injury.

\section{Protection Against Mild TBI}

\section{I: Helmet Standards}

Next to impact avoidance, football helmets are the most important factor in protecting a player from mild TBI. In 1973, the NOCSAE established standards for the impact performance of football helmets. ${ }^{9}$ The NOCSAE standard limited the SI, which is based on resultant head acceleration. All new football helmets available for use in high school and college football were then certified to the NOCSAE standard, and the wearing of such helmets was made mandatory for college players in 1978 and for high school players in 1980.

The certified helmets cut the SI score by half compared with the headgear worn before the establishment of the standard. By 1980, significant reductions in injuries were observed after the voluntary adoption of the standards by helmet manufacturers. The injury reduction was believed to be the result of the helmet design changes, which targeted serious brain injuries such as brain contusion. Despite the improvement of helmet design for the prevention of serious brain injury, little was known regarding the effectiveness of football helmets in protecting against mild TBI at the time of the initial research conducted by 
the NFL's Committee on Mild Traumatic Brain Injury. Therefore, the Committee planned a series of research projects aimed at defining the biomechanics of concussive impacts in professional football.

After consideration of various alternatives, the effort focused on analyses of game videos of plays that had resulted in concussions. Experts in biomechanics proposed that, with multiple views of the impact and line markings on the field, the direction and speed of concussive impacts could be determined. Cinematographic analysis methods were developed to determine the actual speed at which players were moving before colliding (Fig. 1). This would allow laboratory reconstructions (reenactments) of the game impacts by using instrumented test dummies to simulate the helmeted players. The reenactments closely matched the situations on the field.

Reconstruction of the game impacts involved two Hybrid III anthropometric test devices. Two high-speed videos recorded head kinematics in the reconstruction. The cameras were positioned identically to the views from the game video to allow one-to-one comparison (Fig. 2). With the aid of transducers placed in the head of the dummy, the translational and rotational accelerations of the head could be determined in concussive and noninjurious impacts. Matching the available on-field injury video to clinically confirmed mild TBI made determination of an appropriate "event" possible and verifiable. All mild TBI events in the players were examined, confirmed, and recorded by NFL team physicians.

When a mild TBI occurred on the field, it was evaluat- ed by a physician and athletic trainer, who completed forms describing the impact and the injury. Mild TBIs were also reported to a biomechanical engineering group contracted to analyze and reconstruct game impacts. Television network tapes of games were obtained from the NFL and analyzed.

The most striking observation in this study is that concussion in professional football involves a mean impact velocity of $9.3 \mathrm{~m} / \mathrm{second}(20.8 \mathrm{mph})$ and a head velocity change of $7.2 \mathrm{~m} / \mathrm{second}$ (16.1 mph). These are exceptionally high velocities and accelerations and long durations. Automotive crashes typically involve impact durations of less than $6 \mathrm{msec}$ for head impacts with vehicle rails, pillars, and structures. The NFL results established new information on tolerances in the 15-msec range; there had been a virtual absence of scientific data on human tolerance. The NFL reconstruction data also supported a value of 70 to $75 \mathrm{G}$ for concussion in padded impacts, which is at the high end of earlier tolerance ranges but is consistent with the Wayne State University concussion tolerance curve. Most important, the initial study demonstrated the strong correlation of concussion with translational acceleration, which should therefore be the primary measure for assessment of the performance of helmet protection systems. ${ }^{10}$

One conclusion of the initial biomechanical study was that the current NOCSAE SI and the more widely accepted Head Injury Criterion are adequate performance measures for helmet standards and that the added complexity of measuring rotational acceleration may not be needed
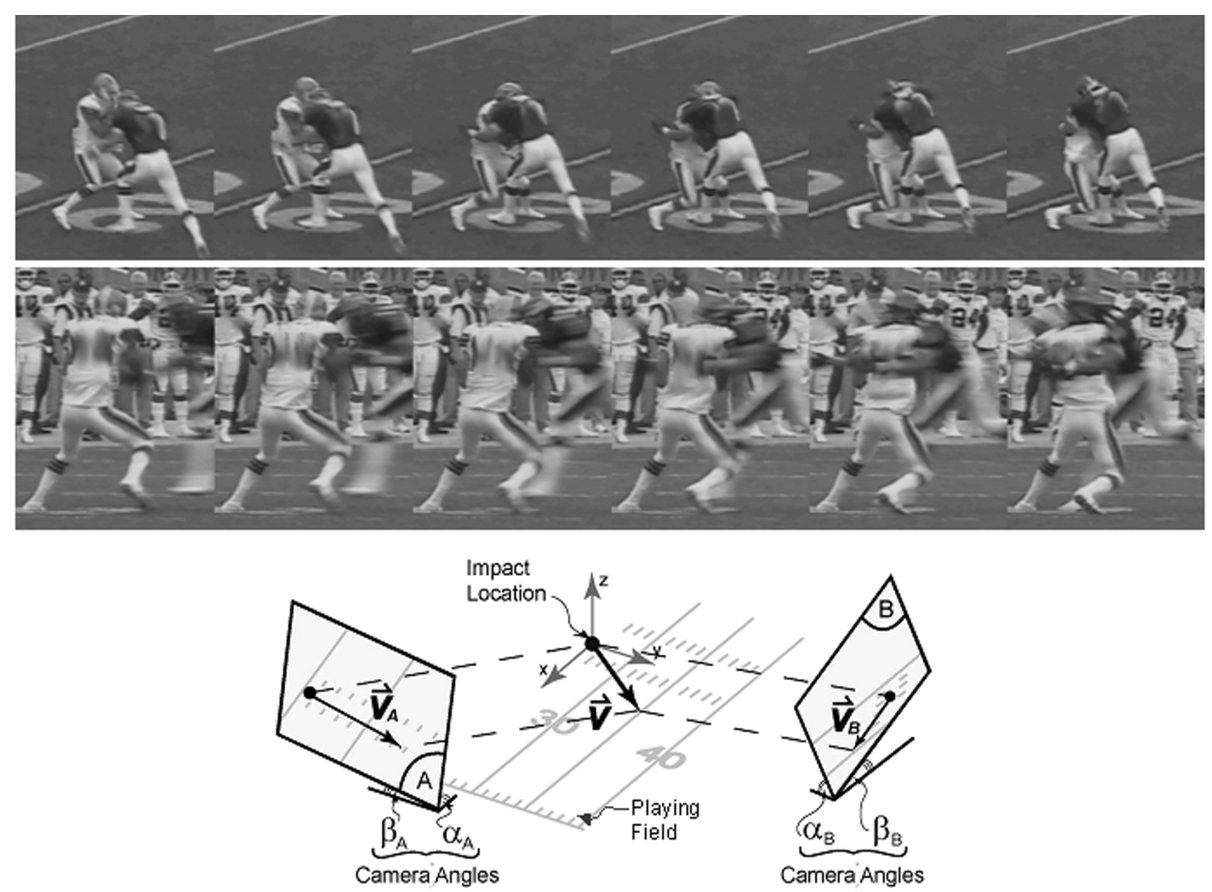

FIG. 1. Still photographs from films showing game action and mathematical calculations of the vector of impacts. The impact velocity of game hits was determined by analysis of two camera views of the collision. Upper: The photos show the impact sequence from two views. Lower: Graph showing the camera locations and the perspective of the two video images of the game impact. The two perspectives are mathematically merged as vectors that change with each time-step of the video. (Reprinted in modified form with permission from Pellman EJ, Viano DC, Tucker AM, Casson IR, Waeckerle JF: Concussion in professional football: reconstruction of game impacts and injuries. Neurosurgery 53: 799-814, 2003.) 


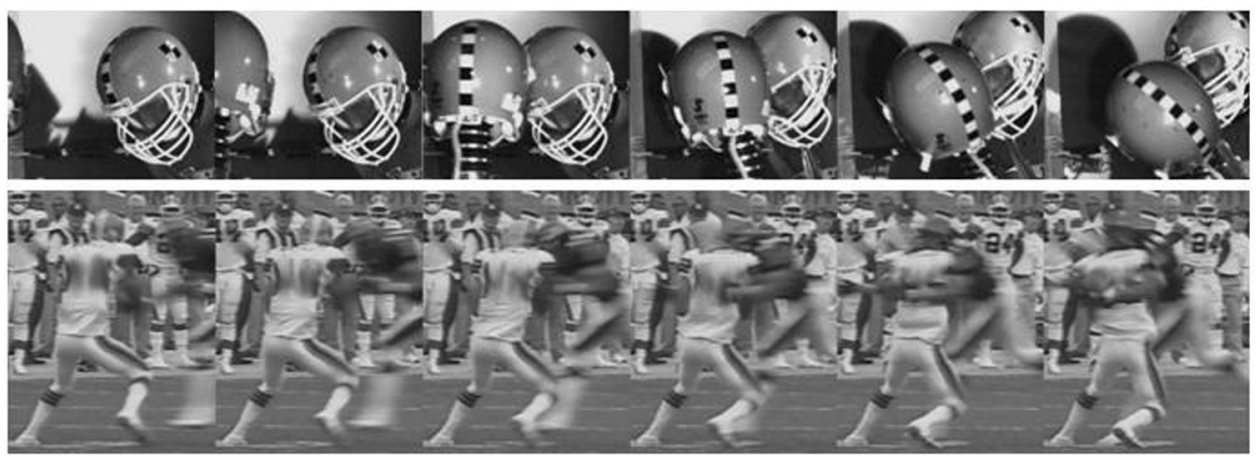

FIG. 2. Comparison of the laboratory reconstruction (upper) and still photos of the game impact (lower) from a case of concussion sustained in an NFL game. Refinements in the test setup were done until the helmet kinematics matched the game impact sequence. (Reprinted in modified form with permission from Pellman EJ, Viano DC, Tucker AM, Casson IR, Waeckerle JF: Concussion in professional football: reconstruction of game impacts and injuries. Neurosurgery 53: 799-814, 2003.)

for an improved or supplemental NOCSAE helmet standard. The results of this study provided a basis for new helmet evaluation methods, new helmet designs, and the prevention of concussions in football.

\section{II: Biomechanical Testing}

It was recognized by the Committee that a greater understanding of the location and direction of helmet impacts was needed to give manufacturers the ability to develop newer, improved mild TBI-resistant helmets. Therefore, NFL game videos were further analyzed for the typical locations of severe helmet impacts in professional football. The magnitude and direction of force causing concussion were determined by the use of selected cases that were reconstructed in laboratory tests.

A request was made to have a biomechanical testing contractor reconstruct the impact in 31 cases by using at least two clear video reviews of the collision. Laboratory

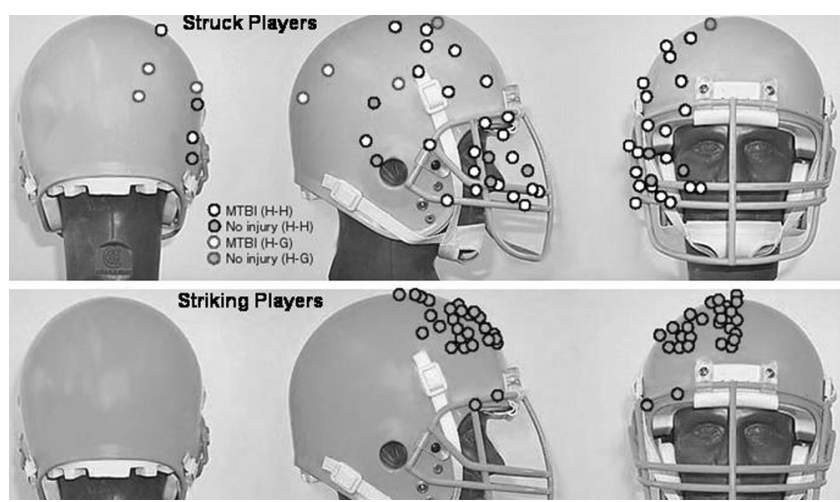

FIG. 3. Upper: Photographs of dummy heads showing location of initial helmet contacts for the struck players. Both concussive and nonconcussive impacts and falls to the ground are shown. Lower: The impact location for the striking players involved no concussions. The impact locations are all shown on the right side of the helmet, although the game impacts occurred on both sides. $\mathrm{H}-\mathrm{G}=$ helmet-to-ground impact; $\mathrm{H}-\mathrm{H}=$ helmet-to-helmet impact; MTBI = mild TBI. (Reprinted with permission from Viano DC, Pellman EJ: Concussion in professional football: biomechanics of the striking player-part 8. Neurosurgery 56: 266-280, 2005.) tests would then be set up to reenact the game impacts with crash dummies and to measure head responses. The reconstruction emphasized helmet-to-helmet and helmetto-ground impacts, because the video of other impacts was more obscured from clear view. Helmet contact of the struck player was categorized by the impact quadrant and head level for helmet contacts.

The study demonstrated the importance of face-mask injuries at an oblique angle, with the majority of contacts occurring below the head's center of gravity. ${ }^{9}$ Another important aspect was that it described the quadrants on the helmet for which future NOCSAE standards may establish performance requirements (Fig. 3). By defining relevant quadrants, greater performance may be ensured over a segment of the helmet in which risks of concussion are higher in professional football, particularly low on the side and back and oblique to the face mask.

The laboratory reconstruction of game impacts provided the Committee with data identifying the location and direction of helmet impacts associated with concussion in NFL players. It also provided unique biomechanical data on head responses associated with concussion. The response data also allowed the determination of injury risk functions for concussion.

Using the Logist function, the probability of concussion $\mathrm{p}(\mathrm{x})$ was related to various biomechanical parameters (x) measured in the reenactment tests by using the following formula: $p(x)=[1+\exp (\alpha-\beta x)]^{-1}$ where $\alpha$ and $\beta$ are parameters fit to the NFL data. The parameters determined for NFL concussion were as follows: $\alpha=2.677$ and $\beta=$ 0.0111 for the Head Injury Criterion; $\alpha=4.678$ and $\beta=0.0573$ for translational acceleration; and $\alpha=5.231$ and $\beta=0.000915$ for rotational acceleration. ${ }^{10}$

\section{III: Head-Down Tackling}

For decades head-down tackling (or so-called spearing) has been a concern because it can result in catastrophic neck injuries in the striking player. The epidemiological and cinematographic analyses of neck injuries have shown that axial loading with flexion or extension causes the majority of cervical fracture-dislocations. This evidence has resulted in rules changes in high school, college, and professional football banning deliberate spearing 
and the use of the top of the helmet as an initial point of contact in a tackle. It was observed that players who suffered concussions were sometimes struck by players who were using head-down tackling techniques. The Committee decided to study the biomechanics of this form of injury both in the striking player ("nonconcussed") and the player who was struck ("concussed").

Once again, game film and video were collected from the NFL and correlated with clinical mild TBI data supplied by each club's team physicians. Laboratory reconstruction was performed using Hybrid III male dummies. In the dummy representing the striking player, a six-axis neck transducer was installed between the head and the top of the neck.

In helmet-to-helmet impacts, the striking player lowers the head, neck, and torso to deliver maximum force to the struck player, whose head and neck resist the impact. ${ }^{15}$ This is the typical situation when the struck player does not see the tackle and does not prepare for the collision. The key to the concussive blow is the head-down position, which involves a $67 \%$ greater mass of the striking player by engaging his torso in the collision. Neck forces couple torso mass into the collision, which contributes to the higher effective mass of the striking player.

The prevention of concussion in the struck player provides another reason, besides preventing neck injuries in the striking one, to enforce rules against head-down tackling or spearing in football. Another means to lower concussion severity may be to reduce the stiffness of the topcrown portion of the helmet and to lower the mass of the helmet, although these changes may be less effective than enforcement of antispearing rules.

\section{IV: Boxing}

Because boxing entails considerable risk of closed head trauma, comparisons are often made between this sport and football regarding mechanisms of injury. The risk of concussion is considerably greater in professional boxing compared with professional football. The clinical picture of more severe brain injury is different in football and boxing. Boxers are much more likely to suffer subdural hematomas and deaths from brain injury than are professional football players. A better understanding of the biomechanics of head responses and mechanisms of brain injury would continue to lay the foundation for better protective headgear for sports.

Eleven Olympic boxers were included in this study. ${ }^{13}$ These athletes were instructed to strike an instrumented Hybrid III head with their gloved fist two times with four different punches (to the forehead and jaw and with a hook and an uppercut). The height and weight of each boxer were measured and anthropometric data for the dominant hand were collected to allow the effective hand-arm mass to be calculated. Instrumentation was placed in the boxer's clenched hand as well as in the Hybrid III head. A camera recorded the event at a lens speed of 4500 images per second. The punch and head inertial forces were measured.

There were three significant differences noted between the biomechanical forces exerted on the head and brain by boxing punches and the football helmet impacts in the NFL. The boxer's punches resulted in lower translational accelerations in the struck head compared with the football impacts (Fig. 4). The boxer's punch applied a higher moment to the struck head than did the football impacts. This resulted in a higher rotational acceleration in the head that was struck than did the football impacts. Boxers sustain a brain injury by two mechanisms: translational and rotational accelerations of the brain, with a preponderance of the rotational component. Professional football players, on the other hand, sustain mild TBI mostly by translational forces because the shell of the helmet allows the players to slide relative to one another, limiting head rotational accelerations. These differences were further studied using finite element analysis of brain responses. ${ }^{14}$ The localized strains in the brain and different biomechanical inputs help explain the clinical differences between head injuries in boxing and professional football.

Finite element modeling also showed that strains develop late, after the primary impact force, and focus on their response at the midbrain. This study shows a complicated interaction of the head kinematics, detailed geometrical and material properties of the brain, and the role of brain movement and deformation within the skull (Fig. 5).

\section{V: Impact Velocity}

In our earlier studies, we found that concussions in NFL players occur at an impact velocity of $9.3 \pm 1.9 \mathrm{~m} / \mathrm{second}$ $(20.8 \pm 4.2 \mathrm{mph})$ oblique on the face mask, side, and back of the helmet. There is a need for new testing methods to evaluate helmet performance in protecting against impacts causing concussion.

The NOCSAE certifies the helmets used by professional football players. The impact tests provide confidence that protective helmets are effective in reducing life-threatening head injuries. Data collected from the accelerometers used in the NOCSAE head drop test are used to assess the shock-attenuating properties of the helmet based on the head SI, in which the risk of serious head injury is

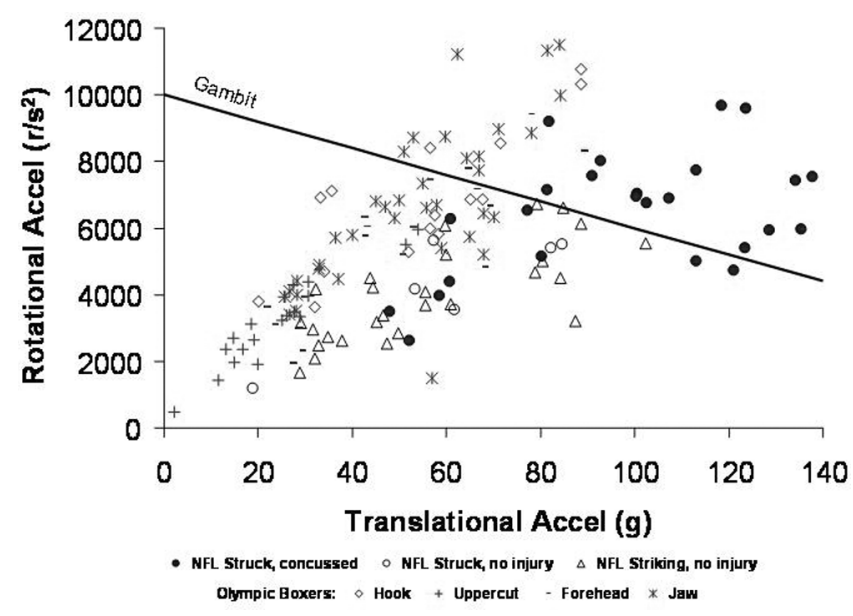

FIG. 4. Scatterplot showing individual data points for translational and rotational acceleration (Accel) of the Hybrid III head for NFL game impacts and four different Olympic boxing punches. (Reprinted with permission from Viano DC, Casson IR, Pellman EJ, Bir CA, Zhang L, Sherman DC, et al: Concussion in professional football: comparison with boxing head impacts-Part 10. Neurosurgery 57: 1154-1172, 2005.) 


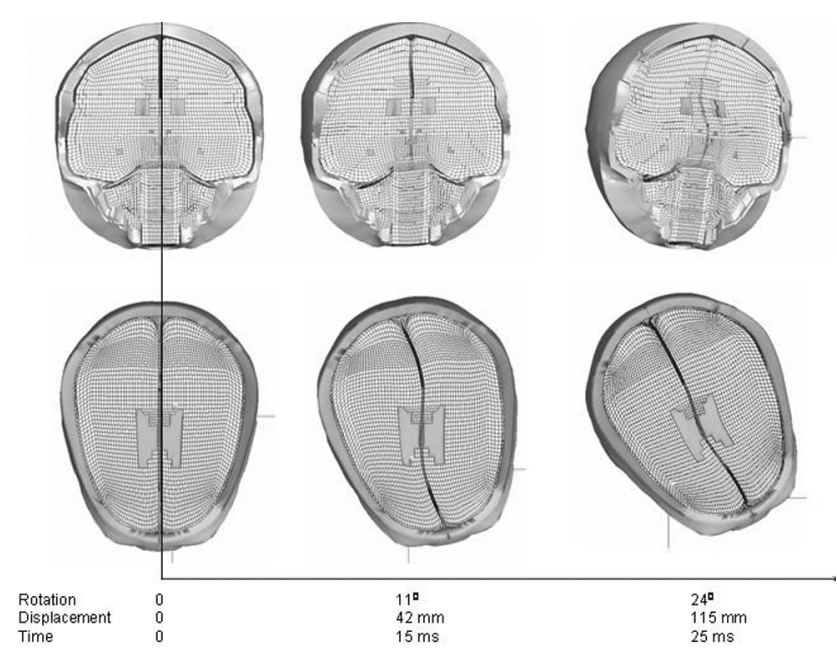

FIG. 5. Anatomical models showing the deformation pattern of the finite element brain from a frontal and superior view of the hemispheres at 0,15 , and $25 \mathrm{msec}$ for one of the NFL concussion cases. The sequence shows the head kinematics and brain deformations. (Reprinted in modified form with permission from Viano DC, Casson IR, Pellman EJ, Zhang L, King AI, Yang KH: Concussion in professional football: brain responses by finite element analysis-part 9. Neurosurgery 57: 891-916, 2005.)

determined from the SI. This standard does not address helmet performance in reducing the risk of concussion.

It was believed by the members of the committee that the previous NFL mild TBI research findings would allow a recommendation to be made for a new methodology for testing helmets to reduce the risk of concussions. ${ }^{11}$ The initial approach involved pendulum impactors that were used to simulate 7.4 and $9.3 \mathrm{~m} / \mathrm{second}$ impacts causing concussion in NFL players. A helmet was placed on an instrumented Hybrid III head that was supported on the neck, which was fixed to a sliding table for frontal and lateral impacts. The testing evolved to a linear pneumatic impactor, which gives better control and a broader speed range for helmet testing. The NOCSAE has prepared a draft supplemental helmet standard for the 7.4- and 9.3$\mathrm{m} /$ second impacts evaluated using the new impactor. The proposed NOCSAE standard is the first to address helmet performance in reducing the risk of concussion.

\section{VI: Performance of the Newer Helmets}

The new understanding of the biomechanics of concussion in NFL players has enabled football helmet manufacturers to make design changes, for the first time, specifically to reduce the risk of mild TBI. The NFL testing techniques addressing concussion were shared previously with the helmet manufacturers and NOCSAE. The Adams USA Pro Elite, Riddell Revolution, and Schutt Sport Air Varsity Commander and DNA helmets are examples of headgear designed using the new information. Using the new mild TBI testing methodology, the Committee believed it would be useful to test the performance of newer helmets in reconstructed game impacts to compare them with a more standard VSR-4 football helmet.

The aim of this most recent study ${ }^{16}$ was to investigate the performance of newer football helmets under conditions causing concussion in NFL players. Ten cases of NFL game concussions were selected for reenactment testing with newer helmets to investigate the equipment's effectiveness in reducing the risk of concussion. The range of impact speed was between 7.4 and $11.2 \mathrm{~m} / \mathrm{second}$ for eight cases of helmet-to-helmet impacts. This was within one standard deviation of the average condition for concussion in the NFL. The two head-to-ground impact cases averaged $7.2 \mathrm{~m} / \mathrm{second}$. For each case of helmet-to-helmet impact, the striking and struck dummies were oriented to match the original laboratory reconstructions of NFL players' concussions. Verification tests ensured that the 10 reconstructed impacts from NFL games were set up similarly to the original testing. Identification on all helmets was obscured, and random tests were conducted.

Testing revealed that newer football helmets reduce concussion risks in collisions that were representative of NFL player experiences. Depending on the biomechanical response, the reductions are in the range of a 10 to $20 \%$ lower risk of concussion. The newer headgear reduces concussion risk by using thicker and more energy-absorbing padding on the side and back of the helmets and around the ears. This demonstrates an encouraging trend with the newer headgear; and we expect additional progress. The tests should help NOCSAE in its effort to finalize new helmet standards for preventing concussions.

\section{Injury Collection and Data Analysis}

\section{I: Prevalence of Mild TBI}

Mild TBI is a major public health problem in the US, with an estimated annual incidence of 160 to 375 cases per 100,000 persons per year. Officials at the Centers for Disease Control and Prevention have estimated that the number of mild TBIs has reached 300,000 cases per year in all sports. In an attempt to better understand mild TBI in the NFL, the Committee supervised prospective collection of data on this condition in NFL players from 1996 to 2001.

All data were collected using standardized forms, and the information was assessed in a blinded fashion. In all, 787 game-related cases (1913 games) were reported, and all players were examined by team physicians, with information reported on player position, type of helmet, symptoms, medical actions, and playing days lost. ${ }^{5}$ All patients were evaluated by physicians immediately after the injury and underwent follow-up physician evaluations until they returned to play. Forms were completed by the physicians, which increased the medical validity and reliability of the information collected. Because the Committee did not mandate case management for mild TBI, the patients' medical course reflects the true natural history of mild TBIs among professional football players during this 6-year period.

Mild TBIs are relatively common injuries sustained by professional football players. The data indicate that quarterbacks, wide receivers, defensive backs, and specialteam players on kicking units are more likely to sustain these injuries than are offensive and defensive linemen (Table 1). The clinical information helped validate the biomechanical data on professional football-related mild TBIs derived from the earlier studies.

The most common initial symptoms for players who sustained concussions were headaches, dizziness, memory 
TABLE 1

Incidence of mild traumatic brain injury according to player position in National Football League games

\begin{tabular}{|c|c|c|c|c|}
\hline Position & No. of Cases & Incidence $(\%)$ & No. of Game Positions & Risk per 100 game-positions \\
\hline \multicolumn{5}{|l|}{ High Risk } \\
\hline \multicolumn{5}{|l|}{ Offensive } \\
\hline Quarterback & 62 & $7.9 \%$ & 3,826 & $1.62(1.22,2.02)$ \\
\hline Wide Receiver & 94 & $11.9 \%$ & 7,652 & $1.23(0.98,1.48)$ \\
\hline Tight End & 36 & $4.6 \%$ & 3,826 & $0.94(0.63,1.25)$ \\
\hline Running Back & 69 & $8.8 \%$ & 7,652 & $0.90(0.69,1.11)$ \\
\hline \multicolumn{5}{|l|}{ Defensive } \\
\hline Secondary & 143 & $18.2 \%$ & 15,304 & $0.93(0.78,1.08)$ \\
\hline \multicolumn{5}{|l|}{ Moderate Risk } \\
\hline \multicolumn{5}{|l|}{ Offensive } \\
\hline Offensive Line & 56 & $7.1 \%$ & 19,130 & $0.29(0.21,0.37)$ \\
\hline \multicolumn{5}{|l|}{ Defensive } \\
\hline Linebacker & 52 & $6.6 \%$ & 11,478 & $0.45(0.33,0.57)$ \\
\hline Defensive Line & 67 & $8.5 \%$ & 15,304 & $0.44(0.34,0.54)$ \\
\hline \multicolumn{5}{|l|}{ Special Team } \\
\hline Return Ball Carrier & 22 & $2.8 \%$ & 3,826 & $0.58(0.34,0.82)$ \\
\hline Kick Unit & 131 & $16.6 \%$ & 38,260 & $0.34(0.28,0.40)$ \\
\hline \multicolumn{5}{|l|}{ Low Risk } \\
\hline \multicolumn{5}{|l|}{ Special Team } \\
\hline Punter & 7 & $0.9 \%$ & 3,826 & $0.18(0.05,0.31)$ \\
\hline Return Unit & 33 & $4.2 \%$ & 38,260 & $0.09(0.06,0.12)$ \\
\hline Kicker, FGA & 1 & $0.1 \%$ & 3,826 & $0.03(-0.02,0.08)$ \\
\hline Kicker, PAT & 1 & $0.1 \%$ & 3,826 & $0.03(-0.02,0.08)$ \\
\hline Holder & 1 & $0.1 \%$ & 3,826 & $0.03(-0.02,0.08)$ \\
\hline Unknown/Undesignated & 12 & $1.5 \%$ & & \\
\hline Total & 787 & $100 \%$ & & 8.08 \\
\hline
\end{tabular}

aRisk per 100 game-positions is the number of concussions divided by the number of times the position was played during the observed period of 3,826 games, multiplied by 100 . Values in parentheses are $95 \%$ confidence intervals. FGA = field goal attempt; $\mathrm{PAT}=$ point after touchdown. Risk strata are approximate, because there is some overlap of confidence intervals.

(Table reproduced with permission from Pellman EJ, Powell JW, Viano DC, Casson IR, Tucker AM, Feuer H, et al: Concussion in professional football: epidemiological features of game injuries and review of the literature-part 3. Neurosurgery 54:81-96, 2004.)

problems, cognitive problems, and somatic complaints. Headaches were observed in 55\% (95\% CI 51.5-58.5\%) of NFL players who suffered concussions. ${ }^{5}$ For the great majority of these players, mild TBIs did not cause prolonged disability or prolonged absence from play. In the NFL, $56.5 \%$ of players with concussions returned to play on the day of the injury and $97.1 \%$ returned to play by Day 9 after the injury. Only $2.9 \%$ of the players missed more than 9 days before returning to play. This indicates that most mild TBIs sustained in the NFL are self-limiting and that players recover fully and spontaneously in a short time. Because a significant percentage of players returned to play in the same game and the overwhelming majority of players with concussions were kept out of football-related activities for less than 1 week, it can be concluded that mild TBIs in professional football are not serious injuries.

Only 9.3\% (95\% CI 7-11.6\%) of the NFL players experienced an LOC as a result of severe concussive head impacts (58 of 623 reported cases of mild TBI). It is important for all physicians who care for athletes with head injuries to know that most of the concussions they treat are not associated with LOC, and that when this symptom occurs it is for a relatively short duration.

\section{II: Repeated Mild TBI}

Despite the findings and conclusions in the preliminary clinical study, questions remained concerning NFL play- ers who suffered repeated mild TBIs. Physicians have been concerned for many years about the possible deleterious effects of multiple concussions on the brains of athletes. ${ }^{8}$ In this study, data on 887 concussions sustained in practices and games involving 650 players from all 30 NFL teams between 1996 and 2001 were prospectively collected and analyzed. A total of 160 players experienced repeated injury, with 51 suffering three or more concussions during the study period. The median time between injuries was 374.5 days, with only six concussions occurring within 2 weeks of the initial injury. Repeated concussions were more prevalent in the secondary, the kick unit on special teams, and in wide receivers.

There have been reports in which researchers have concluded that there may be an increased risk of repeated concussive injuries, and there may be a slower recovery of neurological function after repeated concussions in those who have a history of previous ones. The results of this study in professional football players do not support that conclusion. Although approximately one half of players returned to play during the same game or practice session, and approximately $90 \%$ returned within 1 week, recurrent injury caused by an increased vulnerability in the immediate postconcussion period does not seem to be a factor in professional football players.

No cases of "second-impact syndrome" were detected during the 6-year period of this NFL study. There were no deaths, prolonged comas, or evidence of diffuse cerebral 
edema in any player. Furthermore, there have been no case reports of second-impact syndrome in the history of the NFL. It is possible that this syndrome does not truly exist in this population of athletes. Many of the mild TBI guidelines have established exclusion periods based at least partially on the belief that everyone who experiences a symptomatic mild TBI is at risk for the development of second-impact syndrome. The absence of the syndrome in this patient population supports the suggestion that such arbitrary return-to-play guidelines may be too conservative for professional football.

Another often-expressed concern underlying the development of mild TBI guidelines is the occurrence of chronic brain damage as a result of multiple head injuries. A recent letter to the editor in Neurosurgery addressed the case of an NFL player who was alleged to have died of complications of chronic traumatic encephalopathy, underscoring this concern. ${ }^{1}$ Chronic traumatic encephalopathy in boxers is a well-accepted and documented clinical and pathological syndrome. The clinical features include a combination of cerebellar, extrapyramidal, and pyramidal dysfunction, along with cognitive and personality changes. In the NFL study, none of these features was identified in any player, including those with repeated injury. There were no signs of chronic traumatic encephalopathy in this group of active, contemporary football players.

In players with four or more concussions there was a greater chance of personality change and fatigue, but the number did not reach statistical significance. The incidence of LOC at the time of mild TBI was no different with successive concussions. Overall, however, the signs and symptoms reported in the NFL study were very similar in players with single and multiple mild TBIs. There was no evidence of increased severity of injury in multiple compared with single mild TBI cases.

\section{III: Postconcussion Syndrome}

In the data analysis there were a small number of athletes in whom persistent postconcussion symptoms developed, and these individuals were unable to return to play for an extended period. Often the postconcussion symptoms were seen 1 or more weeks after the injury. The postconcussion syndrome follows head injury that is usually mild, and consists of any combination of the symptoms and signs that occur after mild TBI. The Committee decided to analyze the data obtained in players in whom postconcussion syndrome was diagnosed.?

When does cerebral concussion end and postconcussion syndrome begin? Very few data are available on the evolution of head injury to postconcussion syndrome in athletes. The purpose of this part of the study was to compare the small group of NFL players who did not return for more than 7 days after a mild TBI with the majority of NFL players who do return within 7 days. The 7-day dividing line between the groups does not reflect an arbitrary distinction. Because NFL teams play games once a week, the players in this study cohort all missed at least one game. The NFL teams play only 16 games per season. Therefore, missing one game involves a significant loss of playing time. The study cohort all had significant functional impairment caused by mild TBI.

There were 72 cases with more than 7 days away from play among the 887 cases of mild TBI analyzed between 1996 and 2001. Of these injuries, 38 were single concussions experienced in the study period, eight were the first of repeated concussions, 16 were the second, seven were the third concussion, and so on in the study period. The median duration between the first injury and 7 or more days away from play was 364 days, and the median duration between the last injury and 7 or more days away was 329 days, which is statistically similar.

For the whole sample, there were 650 players who experienced 887 concussions during the study period, and the position they were playing was recorded in this analysis. Individually, the position groups most often associated with loss of 7 or more days are the defensive secondary $(23.6 \%)$, kick unit $(19.4 \%)$, quarterbacks $(12.5 \%)$, and wide receivers $(12.5 \%)$. The fraction of players in a position with 7 or more days away from play compared with all in that group was highest for the quarterback (14.8\%), the return unit on special teams $(11.8 \%)$, and the secondary $(10.8 \%)$, followed by the kick unit $(10.4 \%)$ on special teams. Quarterbacks had the highest odds ratio of 7 or more days away from play with concussion, whereas running backs had the lowest relative risk.

The majority of players with concussions $(88.9 \%)$ are rested, with 7 or more days out, compared with $90.7 \%$ with fewer than 7 days out. Overall, the data show a conservative treatment of concussion. There was no statistical difference in players' treatment in comparisons between the two groups.

Between the initial examination and the first follow-up review, most of the signs and symptoms started to decrease, except for increases in the general category of memory problems, fatigue, irritability, and sleep problems. By the fourth follow-up examination (median 4.7 days), all memory and cognitive problems had cleared. Nevertheless, some players still reported headaches, dizziness, and photophobia. Somatic complaints continued in some players, including personality change and fatigue. By the seventh examination (median 13 days) only headaches remained to clear.

The data analysis allowed the development of profiles for two groups of NFL players with concussions. The first group is the small minority of players who ultimately do not return to play for 7 or more days postinjury. They are more likely to experience LOC as a result of the head injury, and they are more likely to be hospitalized on the day of the injury. At the time of the initial evaluation, these players have a significantly increased number of the signs and symptoms of mild TBI. On initial examination, they are very likely to have retrograde amnesia, difficulties with immediate recall, and overall difficulties with cognition and general memory.

The results of this study and the previous ones prompted the Committee to perform a critical analysis of the widely promoted guidelines for the evaluation and management of concussion in sport. This 6-year study indicates that no NFL player experienced second-impact syndrome, chronic cumulative injury, or chronic traumatic encephalopathy from repeated injuries. These are a few of the expressed rationales for developing management guidelines. The proponents of these guidelines recommend grading the severity of concussion by a limited number of criteria, such as presence or absence of LOC, post- 
traumatic amnesia at time of injury, confusion, and mental status changes soon after injury. The guidelines then make clinical management recommendations on the basis of the grade of concussion diagnosed using these criteria.

Analysis of the NFL data reveals that there are other prognostic factors of equal importance that are not included in the grading systems. These include photophobia, fatigue, and increased absolute numbers of signs and symptoms. Furthermore, the grading systems do not take into account factors such as the position played by the injured athlete and the type of play during which the injury occurred. In the NFL study we found that the presence of signs and symptoms such as fatigue, sleep disturbance, irritability, and/or cognitive or memory impairments on examination the day after the injury also has significant prognostic usefulness. None of the grading systems incorporate results from examinations performed other than on the day of the injury. Although the grading systems use some important prognostic findings, they are limited in their scope and fail to incorporate a number of other factors that have been demonstrated to be predictors of delayed recovery.

The NFL studies also support the contention that grading concussions immediately after injury is prone to error. A number of players with signs and symptoms suggesting a poor prognosis in fact recovered very quickly and returned to play on the day or within a few days of injury. Conversely, there were two players with minimal signs or symptoms, suggesting a good prognosis, who were ultimately kept out of play for 7 or more days after mild TBI. None of the prognostic factors or combinations of factors was $100 \%$ accurate in predicting recovery.

It followed from this analysis that the current attempts to link prospective grading of concussion symptoms to arbitrary, rigid management decisions are not consistent with scientific data. We believe that if one insists on grading concussion severity, the best way is retrospectively, on the basis of how long it actually takes the player to become asymptomatic, with normal results on neurological examination. It is the recommendation of the NFL's Committee on Mild Traumatic Brain Injury that team physicians treat their players on a case-by-case basis, using their best clinical judgment and basing their decisions on the most relevant, objective medical data obtained.

\section{IV: Neuropsychological Testing in Evaluating Mild TBI}

The development and use of neuropsychological testing in the NFL has been rapid, and it has contributed to the implementation of this testing in other professional sports organizations, including ice hockey, automobile racing, and Australian Rules football. When used in concert with other medical information, neuropsychological test data contribute quantitative information regarding neurocognitive processes, such as attentional, memory, and cognitive processing speed. Neuropsychological testing can provide objective information regarding the recovery process and allows comparisons of the athlete's performance against normative data and the individual's preinjury level of performance.

The NFL's neuropsychological testing program was established as a clinical research program with the goal of investigating the use of such testing to assist team physi- cians in the return-to-play decision. Athletes in the NFL who underwent neuropsychological testing between 1996 and 2001 and who participated in the study were included. ${ }^{4}$ Preseason normative data were collected in 655 NFL athletes. The overall sample of injured players who underwent testing consisted of 143 athletes. This sample represented $22 \%$ of the 650 NFL athletes who experienced 887 concussions during the study period. Because participation in the study was voluntary, not all athletes with mild TBI completed neuropsychological testing.

This study supports previous research that has shown that on-field signs of cognitive impairment, such as amnesia, are useful in determining the severity of brain injury. ${ }^{4}$ Players identified as having cognitive and memory disturbances are likely to show neuropsychological impairments on follow-up testing. Athletes with no clinically recognized cognitive and memory impairments on physician examination did not, as a group, have more subtle changes in cognitive processes that were missed during the sideline clinical examination. This suggests that the on-field evaluation by team physicians is effective with regard to the identification of cognitive and memory impairments immediately after an injury.

On review of the data, the fact that there were no overall significant differences in test results between a group of injured NFL athletes who had previously undergone baseline neuropsychological testing suggests that NFL athletes with mild TBIs recover quickly after injury (Fig. 6). In contrast to previous studies in which cognitive difficulties lasting 1 week or more were suggested, NFL athletes demonstrated generally intact performance within several days relative to baseline performance levels.

The issue of the potential cumulative effects of sportsrelated mild TBI has been a particularly controversial one, and many studies have offered different views on the significance of multiple injuries. In this study we did not find a pattern of worse neuropsychological test scores in a group of professional athletes who received close followup care for 6 years. We also did not find worse neuropsychological performance in NFL athletes who were held back from play for 7 or more days compared with a group who returned within 1 week. It is noteworthy that in this group that was kept from play for 7 or more days, the results of neuropsychological tests and medical evaluation of cognitive and memory function were normal within several days of the injury. The results of this study indicate no evidence of worsening injury or chronic cumulative effects of multiple mild TBIs in NFL players.

On the basis of this study, the NFL's Committee on Mild Traumatic Brain Injury makes the following recommendations regarding the proper role of neuropsychological testing in the NFL. Neuropsychological testing is a tool that can assist the physician in evaluating and managing mild TBI. It definitely should not be used in isolation and cannot replace and should not be used to replace the clinical judgment of the treating physician in the diagnosis and management of mild TBI. The main value of neuropsychological testing in this setting is its ability to confirm and corroborate the results of clinical and mental status evaluation.

\section{V: The ImPACT Program}




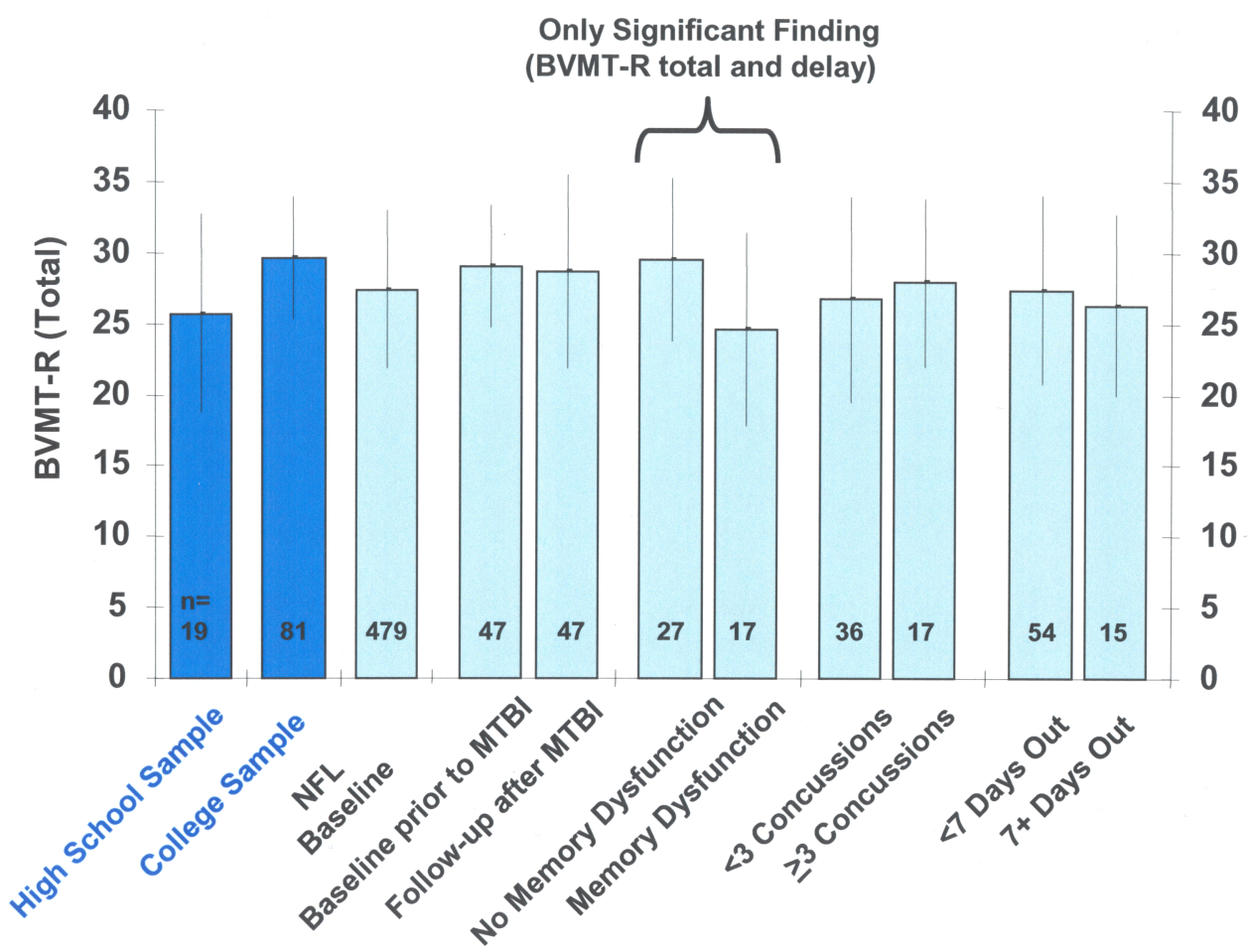

FIG. 6. Bar graph showing the mean and standard deviation in Brief Visuospatial Memory Test-Revised (BVMT-R) (Total) neuropsychological test scores for NFL baselines, players with memory problems, more than three concussions, and out 7 or more days from play. Also shown are data (first two bars) from a sample of high school and college players at Penn State University. (Reprinted with permission from Pellman EJ, Lovell MR, Viano DC, Casson IR, Tucker AM: Concussion in professional football: neuropsychological testing—part 6. Neurosurgery 55: 1290-1305, 2004.)

Despite the initial findings, based on neuropsychological testing, that NFL players had a rapid return to baseline after a mild TBI, the Committee was aware that this was in contrast to several studies that found more long-lasting neuropsychological decrements in high school athletes. Members of the committee decided to perform an additional study, in which professional and younger athletes were compared using the same protocol and identical neurocognitive test battery. ${ }^{3}$

The ImPACT program is a computerized neuropsychological testing instrument used by some within the NFL neuropsychology program. The ImPACT program (version 2.0) consists of six neuropsychological tests designed to target different aspects of cognitive functioning, including attention, memory, processing speed, and reaction time. The results of ImPACT testing on NFL and high school players were compared.

In this study, ${ }^{3}$ we found no significant neurocognitive deficits in the NFL sample within the 1st week postinjury, suggesting that NFL athletes with mild TBIs recover relatively quickly after injury. In contrast, we found residual difficulties in reaction time and memory in the high school sample but not in professional players. This raises the question of differential response to mild TBI in professional and high school athletes.

\section{VI: Return to Play}

There were concerns based on the results of the earlier studies of mild TBI that perhaps some players were being returned to play too soon after injury, thus resulting in more prolonged postconcussion syndrome and perhaps creating the risk of more severe brain injury. The committee therefore decided to do a data analysis on NFL players who returned to play on the same day as their mild TBI. ${ }^{6}$

In the NFL players studied between 1996 and 2001, there were $135(15.2 \%)$ who returned to play immediately after mild TBI and $304(34.3 \%)$ who rested and returned to the same game after concussion. There were few differences in the player position or team activity related to the injury or action taken. However, players who suffered concussions and returned to the same game had fewer initial signs and symptoms than those who were removed from play.

Widely used concussion management guidelines state that athletes can return to play on the day of the injury if they become asymptomatic and if results of examinations performed within 15 minutes of their injury are normal. In the NFL database, $41 \%$ of players returned to the same game either immediately or after resting more than 15 minutes. Of those who returned immediately, $17.9 \%$ were out more than 15 minutes, and $51.7 \%$ of those who rested and returned were out for more than 15 minutes. The data showed no increased risk of repeated mild TBI, prolonged postconcussion syndrome, delayed return to play ( $\geq 7$ days out), second-impact syndrome, or catastrophic intracranial event. The NFL experience thus supports the suggestion that players who become asymptomatic and have normal results on examinations performed at any time after injury, while the game is still in progress, have 
been and can continue to be safely returned to play on that day. The data also support the proposition that players who experienced LOC had no increased risk of repeated mild TBI or prolonged postconcussion syndrome compared with other players.

The results of this study indicate that many NFL players can be safely allowed to return to play on the day of the injury after sustaining a mild TBI. These players had to be asymptomatic, with normal results on clinical and neurological examinations, and be cleared by a knowledgeable team physician. There were no adverse effects, and the results once again are in sharp contrast to the recommendations in published guidelines and the standard of practice of most college and high school football team physicians. This data analysis was performed on information obtained in adult professional football players, and these findings are not meant to be carried over to any other patient population, including high school and college football players.

\section{Conclusions}

The NFL study was conducted to increase the scientific information available to physicians and sports professionals based on prospective clinical information and contemporary, "real-time" biomechanical data. The NFL's Committee on Mild Traumatic Brain Injury is currently supervising work on an animal model of mild TBI, mouthpieces, and studies of retired players that we hope will continue to add information and shed light on the complicated clinical syndrome of mild TBI in athletes.

\section{References}

1. Casson IR, Pellman EJ, Viano DC: Chronic traumatic encephalopathy in a National Football League player. Neurosurgery 58: E1003, 2006 (Letter)

2. Pellman EJ: Background on the National Football League's research on concussion in professional football. Neurosurgery 53:797-798, 2003

3. Pellman EJ, Lovell MR, Viano DC, Casson IR: Concussion in professional football: recovery of NFL and high school athletes assessed by computerized neuropsychological testing - part 12 . Neurosurgery 58:263-274, 2006

4. Pellman EJ, Lovell MR, Viano DC, Casson IR, Tucker AM: Concussion in professional football: neuropsychological test- ing-part 6. Neurosurgery 55:1290-1305, 2004

5. Pellman EJ, Powell JW, Viano DC, Casson IR, Tucker AM, Feuer $\mathrm{H}$, et al: Concussion in professional football: epidemiological features of game injuries and review of the literaturepart 3. Neurosurgery 54:81-96, 2004

6. Pellman EJ, Viano DC, Casson IR, Arfken C, Feuer H: Concussion in professional football: players returning to the same game-part 7. Neurosurgery 56:79-92, 2005

7. Pellman EJ, Viano DC, Casson IR, Arfken C, Powell J: Concussion in professional football: injuries involving 7 or more days out-part 5. Neurosurgery 55:1100-1119, 2004

8. Pellman EJ, Viano DC, Casson IR, Tucker AM, Waeckerle JF, Powell JW, et al: Concussion in professional football: repeat injuries-part 4. Neurosurgery 55:860-876, 2004

9. Pellman EJ, Viano DC, Tucker AM, Casson IR: Concussion in professional football: location and direction of helmet impacts-part 2. Neurosurgery 53:1328-1341, 2003

10. Pellman EJ, Viano DC, Tucker AM, Casson IR, Waeckerle JF: Concussion in professional football: reconstruction of game impacts and injuries. Neurosurgery 53:799-814, 2003

11. Pellman EJ, Viano DC, Withnall C, Shewchenko N, Bir CA, Halstead PD: Concussion in professional football: helmet testing to assess impact performance-part 11. Neurosurgery 58: 78-96, 2006

12. Tagliabue P: Tackling concussions in sports. Neurosurgery 53: 796, 2003 (Editorial)

13. Viano DC, Casson IR, Pellman EJ, Bir CA, Zhang L, Sherman DC, et al: Concussion in professional football: comparison with boxing head impacts-part 10. Neurosurgery 57:1154-1172, 2005

14. Viano DC, Casson IR, Pellman EJ, Zhang L, King AI, Yang $\mathrm{KH}$ : Concussion in professional football: brain responses by finite element analysis-part 9. Neurosurgery 57:891-916, 2005

15. Viano DC, Pellman EJ: Concussion in professional football: biomechanics of the striking player-part 8. Neurosurgery 56:266-280, 2005

16. Viano DC, Pellman EJ, Withnall C, Shewchenko N: Concussion in professional football: performance of newer helmets in reconstructed game impacts-Part 13. Neurosurgery 59:591-606, 2006

Manuscript received June 22, 2006.

Accepted in final form October 2, 2006.

Address reprint requests to: David C. Viano, Dr. Med., Ph.D, ProBiomechanics, 265 Warrington Road, Bloomfield Hills, Michigan 48304. email: dviano@ comcast.net. 